\title{
A systematic evaluation of recurrent miscarriage cases and associated risk factors - a hospital based observational study in North India
}

\author{
Sukhbir Pal Kaur, Susmita Sharma, Gaganlata \\ Corresponding author: Dr. Susmita Sharma, Professor, Department of Obstetrics and \\ Gynaecology, Adesh Medical College and Hospital, Mohri, Shahbad, Haryana, India; \\ Email : sushmitasharma28@gmail.com
}

Distributed under Attribution-Non Commercial - Share Alike 4.0 International (CC BY-NC-SA 4.0)

\begin{abstract}
Background: Recurrent miscarriages have different underlying aetiologies due to which single common treatment protocol is not possible. Thus evaluation of couples for underlying pathologies for recurrent miscarriages is essential to make appropriate treatment plan. Objectives: The aim of this study was to find out common causes of recurrent miscarriages in North Indian population. Material and methods: This was an observational study conducted in a tertiary care hospital in North India. A total of 53 couples presenting with history of three or more recurrent miscarriages were recruited in the study. Relevant history, examination findings and baseline investigations were noted. Specific investigations were done according to findings. Cases were stratified on the basis of maternal age, number of miscarriages and type of aborters (primary/secondary). Results: A probable cause of recurrent miscarriages could be found in $60.4 \%$ of cases. In $13.2 \%$ cases co-existing aetiologies were found. Among known causes, most frequent finding was infections contributing to $20.76 \%$ cases, with CMV being the most prevalent $(11.32 \%)$. Autoimmune pathology was found in $18.86 \%$, with $15.1 \%$ cases with primary antiphospholipid syndrome and $3.8 \%$ with systemic lupus erythematosus. Uterine abnormalities were found in $11.3 \%$ with majority having cervical incompetence $(5.66 \%)$ followed by bicornuate uterus $(3.8 \%)$. Infections and endocrine abnormalities were more common in early miscarriages $(<10$ weeks) whereas anatomical defects were significantly higher at later gestations (>14weeks) and mostly among secondary aborters. Diabetes was detected in $3.8 \%$, luteal phase defect in $9.43 \%$, thyroid abnormality in 1.9\%, parental chromosomal abnormalities in 3.8\% of cases. Conclusion: In our population, prevalence of infections is higher and contribution of autoimmune, anatomic and endocrine factors is also significant. This can help us formulate local cost-effective protocols for evaluation and treatment of recurrent miscarriages.
\end{abstract}

Keywords: Habitual abortion, recurrent miscarriages, recurrent abortion.

Few clinical situations are as exasperating for patients and clinicians as recurrent miscarriages. Recurrent miscarriage is a heterogeneous condition for which various aetiologies have been proposed. In literature, the main causes for recurrent miscarriages have been foetal chromosomal or genetic aberrations, parental chromosomal abnormalities, maternal thrombophilias, infections, autoimmune diseases, endocrine diseases, and anatomic abnormalities. ${ }^{1-4}$

As the aetiologies differ, no single treatment is applicable to all and hence evaluation plays an important role in planning individualized treatment. Under ideal conditions, all pregnancy losses should be evaluated fully, however, high costs and limited resources make it important to follow certain selection criteria or protocols.

Various studies have been done in the developed world and guidelines published according to their environmental and economic conditions. India has different socio-economic conditions and environmental conditions and it seems

Received: $4^{\text {th }}$ October 2020, Peer review completed: $12^{\text {th }}$ February 2021, Accepted: $3^{\text {rd }}$ March 2021.

Kaur SP, Sharma S, Gaganlata. A systematic evaluation of recurrent miscarriage cases and associated risk factors - a hospital based observational study in North India. The New Indian Journal of OBGYN. 2022; 8(2): 262 - 68. 
reasonable to presume that certain aetiologies like infections may be more common here. With this background we planned this study to investigate cases with recurrent miscarriages to identify the underlying causes and find the impact of different causes contributing to it.

\section{Material and methods}

This study was an observational study conducted in a tertiary care hospital in North India from December, 2002 to January, 2004. Couples presenting with three or more recurrent miscarriages before the period of viability i.e. 24 weeks of pregnancy were included for evaluation of cause of miscarriage. Patients with ectopic pregnancies and IVF failure were excluded. Relevant history, examination findings and investigation reports were noted on a structured proforma. Reports of investigations like complete hemogram, blood sugar levels, thyroid function tests, USG pelvis and specific tests like antiphospholipid antibodies, LH/FSH assays, culture sensitivity tests, TORCH titre, parental karyotypes, HSG to exclude intracavitary pathologies like intrauterine adhesions, anatomic malformations, cervical incompetence and hysteroscopy findings were noted. Clearance from institutional ethical committee was taken. Written informed consent was obtained from all patients under study. For analysis, cases were stratified on the basis of maternal age, number of miscarriages and type of aborters [primary (with no children)/ secondary (abortion subsequent to having children or stillbirth)].

Student's ' $\mathrm{t}$ ' test was used to test significant difference between means of two groups. A $P$ value of less than 0.05 was taken as significant. ' $z$ ' test was used to find out significant difference between two proportions.

\section{Results}

In our hospital, $14.3 \%$ of total obstetric population reported with miscarriages and $2.2 \%$ had history of recurrent miscarriages. 63 couples were initially recruited in the study over a period of two years. Five cases were excluded from study due to incomplete data and two were lost to follow-up. In three cases, no growth was available on lymphocyte culture for karyotype and no further samples could be taken. Hence, a total of 53 couples were included in study for final evaluation. The details of study population and various etiological factors responsible for recurrent abortions

\begin{tabular}{|c|c|c|c|}
\hline Observations & Number & $\begin{array}{l}\text { Exclusive } \\
\text { aetiology }\end{array}$ & $\begin{array}{l}\text { Co-existent factors } \\
\text { present* }\end{array}$ \\
\hline Total no of cases & 53 & & \\
\hline Mean maternal age in years (range) & $29.7 \pm 4.52(21-40)$ & & \\
\hline Mean paternal age in years (range) & $32.47 \pm 4.61(24-45)$ & & \\
\hline Primary aborters, $\mathrm{n}(\%)$ & $23(43.4 \%)$ & & \\
\hline Secondary aborters, n (\%) & $30(56.6 \%)$ & & \\
\hline Infections, $\mathrm{n}(\%)$ & $11(20.76 \%)$ & $9.46 \%$ & $11.3 \%$ \\
\hline Endocrine abnormality, n (\%) & $10(18.86 \%)$ & $17 \%$ & $1.86 \%$ \\
\hline Autoimmune abnormality, n (\%) & $10(18.86 \%)$ & $11.4 \%$ & $7.5 \%$ \\
\hline Anatomic abnormality, n (\%) & $6(11.32 \%)$ & $9.4 \%$ & $1.9 \%$ \\
\hline Cytogenetic abnormality, n (\%) & $2(3.8 \%)$ & & \\
\hline Unknown cause, $\mathrm{n}(\%)$ & $21(39.6 \%)$ & & \\
\hline
\end{tabular}
singularly or in combination are shown in table 1.

The number of miscarriages per couple varied from 3 to 11 with mean of $4.06 \pm 1.68$ miscarriages. In women less than $30 \mathrm{yrs}$ of age this was $3.6 \pm 1.36$ while in those above $30 \mathrm{yrs}$ it was $4.28 \pm 3.57$ miscarriages. The distribution of miscarriages in groups stratified by age was found to be $9.4 \%$ in $20-24 \mathrm{yrs}$, $32.1 \%$ in $25-29 \mathrm{yrs}, 41.5 \%$ in $30-34 \mathrm{yrs}, 15.1 \%$ in $35-39 \mathrm{yrs}$ and $1.9 \%$ in $40-44 y r s$. Previous three miscarriages were

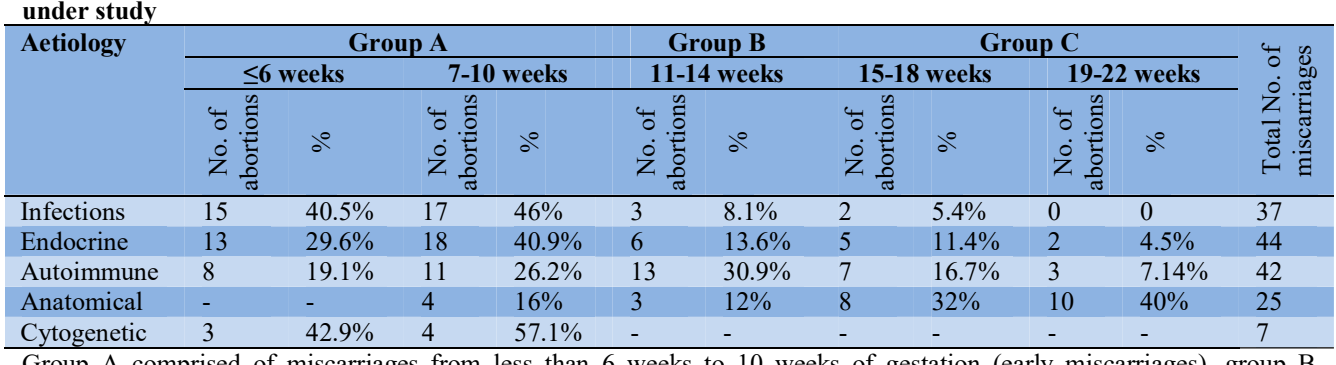
Group A comprised of miscarriages from less than 6 weeks to 10 weeks of gestation (early miscarriages), group B found in $50.9 \%$ of couples, previous four miscarriages in $28.3 \%$ and $20.8 \%$ of cases had five or more miscarriages.

On evaluation, a causative factor could be found in $60.4 \%$ of cases. No definite cause could be assigned to $39.6 \%$ of cases and these were grouped under unknown aetiology. Infections were the most frequent finding (20.76\%). Among infections, Cytomegalovirus (11.32\%) was the most common followed by toxoplasmosis $(9.43 \%)$. There was significant overlap in cases with infections due to presence of coexistent multiple infections (15.1\%).

Co-existing etiologic factors were found in $13.2 \%$ of study population and infections again were the most 
common co- existing factor among groups followed by autoimmune factors.

Uncontrolled diabetes was detected in $3.8 \%$ of cases. All cases of diabetes were insulin dependent. Luteal phase defect was found in $9.43 \%$, polycystic ovaries in $3.8 \%$ and thyroid abnormality in $1.9 \%$. Among autoimmune group, primary antiphospholipid syndrome (primary APS) contributed to $15.1 \%$ of cases and $3.8 \%$ cases were secondary to SLE (Systemic Lupus Erythematosus).

Uterine abnormalities were detected in $11.3 \%$. Cervical incompetence contributed to half of these cases $(5.66 \%)$, followed by bicornuate uterus (3.8\%) and septate uterus $(1.9 \%)$. Anatomical defects were more common in secondary aborters $(7.54 \%)$ as compared to primary aborters $(3.77 \%)$.

Uterine abnormalities were detected in $11.3 \%$. Cervical incompetence contributed to half of these cases $(5.66 \%)$, followed by bicornuate uterus (3.8\%) and septate uterus $(1.9 \%)$. Anatomical defects were more common in secondary aborters $(7.54 \%)$ as compared to primary aborters $(3.77 \%)$.

Couples with chromosomal abnormalities constituted $3.8 \%$ (All detected in female partners and none in male partner). Interstitial deletions on long arm of $3^{\text {rd }}$ chromosome were found in our study.

Samples from abortuses were available for karyotyping in 10 cases, among which 8 could be successfully karyotyped. Findings were noted, but due to small sample size results were excluded from final analysis. Sample size remained small because majority of patients came for evaluation to hospital after they had a miscarriage and very few presented during the process of miscarriage. There was one case of trisomy, one with monosomy 19 and one case with deletion on long arm of X chromosome among tested abortuses. In all these three cases the parental karyotypes were normal.

On comparison of gestational ages at time of miscarriages, infections and endocrine abnormalities were responsible for miscarriages at early gestation $(<10$ weeks) whereas anatomical defects had significantly higher miscarriages at later gestations $(>14$ weeks) with $\mathrm{p}$ - value $<0$. 001. The distribution of number of miscarriages with respect to gestational age at the time of miscarriage and aetiology are shown in table 2 . Previous obstetric history was complicated by stillbirths in $7.6 \%$ and with congenital malformations in $7.6 \%$ of cases.

\section{Discussion}

Recurrent miscarriages were first described in literature as 'Habitual Abortions' by Malpas in $1938 .^{5}$ Later, Hertig and co-workers in their study on hysterectomy specimens of women with proven fertility found that 4 among 8 preimplantation embryos and 9 among 26 implanted embryos were morphologically abnormal. ${ }^{6,7}$

According to RCOG, recurrent miscarriages are loss of three or more consecutive pregnancies before the period of viability. ${ }^{8}$ There is a lack of consensus regarding a universal definition for recurrent miscarriages ever since the concept was first introduced. Subsequently, a number of factors contributing to this entity have been studied and incorporated into evaluation.

Many authorities recommend that evaluation of recurrent miscarriages should be started after two miscarriages. 9, 10 Due to different levels of neonatal care available in developing and developed nations, the period of viability also remain debatable. Williams's obstetrics mentions gestational age of 20 weeks as period of viability whereas RCOG, ESHRE, and SAJOG considers 24 weeks. ${ }^{8-12}$ In this study, we have considered 24 weeks as the period of viability and three or more miscarriages as recurrent miscarriages. A criterion of consecutive or non-consecutive losses does not seem to contribute to aetiology and management of recurrent miscarriages.

Pregnancy loss is recognised in $15-20 \%$ of all pregnancies. ${ }^{4,13}$ Recurrent pregnancy loss affects $1-2 \%$ of all couples trying to conceive, with $80 \%$ of losses occurring in first trimester. ${ }^{14}$ We observed slightly higher percentage of cases with recurrent miscarriages $2.2 \%$ as compared to $1 \%$ by Stirrat ${ }^{2}, 0.8 \%$ by Alberman ${ }^{15}, 0.88 \%$ by Sugiura et $\mathrm{al}^{16}$ and $1.2 \%$ by Pradhan et al. ${ }^{17}$ This can be attributed to the fact that our hospital is a referral centre and total number of cases of miscarriages presenting to our hospital might not be a true representation of miscarriages occurring in community.

The maternal age in our study ranged from 21 to $40 \mathrm{yrs}$ of age with mean age of $29.7 \pm 4.52$ years. Carp et al (2001) in their study on recurrent abortions reported mean maternal age of $31.57 \pm 5.57$ years. ${ }^{18}$ Maternal age is an independent risk factor for miscarriages. A Scottish study reported increased risk after 30years, irrespective of any history of previous miscarriages. ${ }^{19}$ In our study also the mean number of miscarriages above 30 years of age was higher (4.28 \pm 3.57$)$ than those younger than 30 years $(3.6 \pm 1.36)$. Advanced paternal age ( $>40$ years) is also identified as a risk factor ${ }^{20}$, but literature is sparse.

In our study, it was noted that secondary aborters (women with abortions subsequent to having children or stillbirth) had higher incidence of miscarriages at higher gestations ( $>10$ weeks) $(43.6 \%)$ when compared with primary aborters $(23.2 \%)$. 
The anatomical abnormalities seen in our study group comprised of $11.3 \%$ of cases among which, cervical incompetence was most common (5.6\%). Incidence of anatomical defects was also higher in secondary aborters $(7.55 \%)$ and all cases of cervical incompetence $(5.66 \%)$ in our study were found among secondary aborters. Pederson and Pederson (1984) also reported higher rate of cervical incompetence in secondary habitual aborters. ${ }^{1}$ Among mullerian abnormalities, bicornuate was more common finding than septate uterus in our study unlike available literature. ${ }^{21}$ Pederson and Pederson (1984) reported 15.4\% cases with uterine body abnormalities and $12.8 \%$ cases with cervical incompetence. Maione et al (1995) reported 19.5\% cases with uterine abnormalities and $2.4 \%$ with cervical incompetence ${ }^{22}$. Unlike previous studies on this aspect, we did not encounter any case of uterine synechiae or clinically relevant leiomyomas (sub-mucosal $>4 \mathrm{~cm}$ ) that could be ascribed to recurrent miscarriages.

Cytogenetic analysis of parental karyotypes revealed abnormalities in $3.8 \%$ of in our study. Parental chromosomal rearrangements are reported as cause in 3-5\% of couples with recurrent pregnancy loss. ${ }^{12}$ The most common chromosomal abnormality found in parental karyotypes are translocations. Our study revealed structural abnormalities in maternal karyotype in the form of interstitial deletions on long arm of $3^{\text {rd }}$ chromosome. One of the patients showing abnormality in karyotype had two morphologically normal live births followed by four miscarriages, whereas the second case with abnormal karyotype, had one unexplained preterm intrauterine demise, one neonatal demise (with history of cranial abnormality but no records available) followed by three consecutive miscarriages. In literature, deletions on long arm of $3^{\text {rd }}$ chromosome have been reported to cause delayed development, behavioural problems, psychiatric problems, congenital abnormalities ${ }^{23}$ and it also contains gene loci for various inborn errors of metabolism, thrombophilia due to elevated histidine rich glycoprotein ${ }^{24}$ but no direct linkage to recurrent miscarriages is seen in available data. No other structural or numerical abnormality was detected in our study.

Endocrine abnormalities were detected in $18.9 \%$ of cases in our study. Various studies have reported a range from 5\% to $29 \%$ of all abnormalities. ${ }^{4,22,25,26}$ Presence of uncontrolled diabetes in pre-conceptional period with raised HbA1c levels increase the risk of congenital malformations and recurrent abortions. Our study detected $3.8 \%$ cases with insulin dependent diabetes as compared to $1.2 \%$ by Saito in a Japanese study and $2.8 \%$ by Pradhan et al. ${ }^{17}$ Polycystic ovaries may also contribute to pregnancy loss by the effects of insulin resistance, hyperinsulinemia and hyperandrogenemia. ${ }^{27}$ In a study of women with PCOS, Insulin resistance has been observed in $27 \%$ of women with unexplained pregnancy loss. ${ }^{28}$ PCOS was detected in $3.8 \%$ cases in our study. We observed hyperthyroidism in $1.9 \%$ which is slightly lower as compared to $2.8 \%$ by Saito. ${ }^{4}$ Subclinical hypothyroidism with TPO antibodies can also increase risk of recurrent miscarriages. ${ }^{27}$

Another cause ascribed to this entity is luteal phase defect (LPD), in which diagnosis and management is now debatable. Studies reflect incidence of LPD from $12 \%$ to $28 \%$ owing to the variability in the diagnostic methods and criteria. $^{28,29}$ Our study had $9.43 \%$ of cases attributable to LPD. Luteal phase deficiency leads to poor follicular growth, inadequate corpus luteal function and altered endometrial response. LPD can be affected by various factors like hyperprolactinemia, polycytic ovaries, stress, excercise, hypogonadotrophic hypogonadism, poor ovarian reserve and extreme weight loss. $^{27}$ There are inconsistencies in diagnostic methods available and there is paucity of a standard and reproducible test due to which, testing for LPD is not recommended at present. ${ }^{9,30}$ There is insufficient evidence to recommend use of progesterone in patients with recurrent miscarriages with LPD. ${ }^{8,9}$

Autoimmune pathology was noted in $18.86 \%$ of patients in our study with $15.1 \%$ with primary APS and $3.8 \%$ secondary to SLE. This is consistent with the large metaanalysis, where incidence of APS was $15-20 \%$ in cases of recurrent pregnancy loss as compared to $5 \%$ of women without any obstetric complication. ${ }^{31,32}$ According to various reports 7 to $30 \%$ of women with SLE have secondary APS. $^{33,34}$

Infections were the most frequent association in cases of recurrent miscarriages in our study and also the most frequent association in co-existent factors. There is no clear linkage between a long-standing infection and recurrent miscarriages established due to paucity of data due to lack of prospective studies. There is evidence that bacterial vaginosis can lead to second trimester miscarriages. Donders et al, studied 228 cases less than 14 weeks gestation and found increased risk of miscarriage in cases with bacterial vaginosis. $^{35}$. CMV infection was the most common association found with miscarriages in our study followed by Toxoplasma, Rubella and Herpes. CMV can produce both chronic and recurrent maternal infections causing vascular insufficiency and tissue damage resulting in miscarriages. ${ }^{36}$ Immune mediated process by CMV infection can also cause 
The New Indian Journal of OBGYN. 2021 (January-June);8(2)

miscarriages. Re-infection with different strain of CMV in areas with high seroprevalence like India ${ }^{37}$ needs to be studied to find its strength of association to recurrent miscarriages. Other infections studied in relation to poor pregnancy outcome are Gardenella vaginalis, Ureaplasma urealyticum, Mycoplasma Hominis,Toxoplasma gondii, HSV and rarely, syphilis. ${ }^{36}$

Even after extensive work up from available resources, no cause could be ascertained in $39.6 \%$ of patients. In literature, the range of unknown aetiology varies from $15 \%$ to $80 \%{ }^{1,4,22,25,26,38}$ reported as $30 \%$ by Saito $(2009)^{4}, 52.2 \%$ by Maione et al $(1995)^{22}, 37 \%$ by Tho et al $(1979)^{25}, 44 \%$ by Pederson and Pederson (1984). ${ }^{1}$ Thus, counselling the couple before investigating is essential. They should be informed that even if no definite cause can be ascertained in majority of patients the prognosis in such cases is excellent. ${ }^{8}$

Most of the recommendations disapprove of using empirical treatment in such cases as the chance of successful pregnancy without any treatment is high (up to $75 \%$ ). ${ }^{8}$ This might be true for cases where 'chance factor' for miscarriages is there. But there is a small fraction that is genuinely having some pathology which is not discovered yet ${ }^{39}$. It might be influenced by absence of time sensitive tests, limitations of diagnostic modalities available and pathologies which have not been explored yet. There are so many areas where research is still undergoing. The abnormal immunologic reactions, uterine NK cells, ovarian aging and its effects on embryo, DNA fragmentation in sperms, endometrial receptivity issues and many more other undiscovered factors may be underlying in this unknown aetiology group.

The limitations of this study were, the limited funding of the study restricting the chromosomal analysis to conventional metaphase karyotypes and shortcoming like dependence on previous obstetric history told by recall method due to inadequate data in hand held reports in some cases.

From this study, it is evident that further research is needed to fill the gap left by unknown causes of recurrent miscarriages. Genetic screening in such cases may provide with the answer to some of the queries, as diagnosis is improving with invent of newer modalities. Also, it will add to the existing knowledge of genetic basis of this entity. Further research is needed to evaluate causes like infections as there prevalence is considerably higher in our population than western populations and also reevaluate its cause effect relationship.

\section{Conclusion}

In our population, prevalence of infections is higher and contribution of autoimmune, anatomic and endocrine factors is also significant. This can help us formulate local costeffective protocols for evaluation and treatment of recurrent miscarriages.

\section{Conflict of interest: None. Disclaimer: Nil.}

\section{References}

1. Stray Pederson B, Stray Pederson S. Etiologic factors and subsequent reproductive performance in 195 couples with a prior history of habitual abortions. Am J Obstet Gynecol 1984; 148:140-6.

2. Stirrat GM. Recurrent miscarriage. Lancet.1990; 336: 673-5.

3. Khudr G. Cytogenetics of habitual abortion: a review. Obstet Gynaecol Surv. 1974; 29: 299-310.

4. Saito S. The Causes and Treatment of Recurrent Pregnancy Loss. JMAJ. 2009; 52(2): 97-102.

5. Malpas P. A study of abortion sequence. J Obstet Gynecol Br Emp. 1938; 45: 932.

6. Hertig AT, Rock J, Adams EC, et al. Thirty-four fertilized human ova, good, bad and indifferent, recovered from 210 women of known fertility. A study of biologic waste in early human pregnancy. Pediatrics. 1959; 25:202.

7. Hertig AT, Rock J. Searching for early fertilized human ova. Gynecol Invest. 1973; 4:121.

8. Regan L, Backos M, Rai R. The investigation and treatment of couples with recurrent first-trimester and second-trimester miscarriage. RCOG: Green Top Guideline number 17. 2011

9. The ESHRE Guideline group on RPL, Bender AR, Christiansen OB, Elson J, Kolte AM, Lewis S, et al. ESHRE guideline: recurrent pregnancy loss. Hum Reprod Open. 2018; (2): 2018.

10. Practice Committee of American Society for Reproductive Medicine. Definitions of infertility and recurrent pregnancy loss: a committee opinion. Fertil Steril. 2013; 99(1): 63.

11. Cunningham FG, Leveno K.J, Bloom SL, Dashe JS, Spong CY, Hoffman BL, et al. Williams Obstetrics. $25^{\text {th }}$ ed. New York : Mc Graw Hill; 2018. p. 532-69.

12. Niekerk EC van, Siebert I, Kruger TF. An evidence based approach to recurrent pregnancy loss. S Afr J OG. 2013; 19(3): 61-5. 
13. Kutteh WH. Recurrent pregnancy loss: an update. Curr Opin Obstet Gynecol. 1999; 11:435-9.

14. Wilcox AJ, Weinberg CR, O'Connor JF, Baird DD, Schlatterer JP, et al. Incidence of early loss of pregnancy. N Engl J Med. 1988; 319(4):189-94.

15. Alberman E. The epidemiology of repeated abortion. In: Beard RW, Sharp F, editors. Early Pregnancy Loss: Mechanisms and treatment. London: RCOG. 1988. p. 9-17.

16. Sugiura-Ogasawara M, Suzuki S, Ozaki Y, Katano K, Suzumori N, Kitaori T. Frequency of recurrent spontaneous abortion and its influence on further marital relationship and illness: the Okazaki cohort study in Japan. J Obstet Gynaecol Res. 2013; 39:126-31.

17. Pradhan T, Bhavthankar DP. An observational study of causes of recurrent pregnancy loss in rural population. Indian Journal of Basic and Applied Medical Research. 2014; 4(1):70-6.

18. Carp H, Toder V, Aviram A, et al. Karyotype of abortus in recurrent miscarriage. Fertil Steril. 2001; 73: 678-81.

19. Bhattacharya S, Townend J, Bhattacharya S. Recurrent miscarriage: Are three miscarriages one too many? Analysis of a Scottish population- based database of 151,021 pregnancies. Eur J Obstet Gynecol Reprod Biol. 2010; 150: 24-7.

20. Sharma R, Agarwal A, Rohra VK, Assidi M, AbuElmagd M, Turki RF. Effects of increased paternal age on sperm quality, reproductive outcome and associated epigenetic risks to offspring. Reprod Biol Endocrinol. 2015; 13:35.

21. Jaslow CR. Uterine factors. Obstet Gynaecol Clin N Am. 2014; 41: 57-86.

22. Maione S, Lamberti L, Alovlsi C, Armellio F. Retrospective study of couples with history of recurrent spontaneous abortions. Acta Eur Fertil.1995; 26(3): 95-100.

23. Alvarado M, Bocian M, Walker AP. Interstitial deletion of long arm of chromosome 3: case report, review and definition of a phenotype. Am J Med Gen.1987; 27: 781-6.

24. Naylor SL, Garcia DK. Chromosome 3 Encyclopedia of life sciences. New Jersey: John Wiley \& Sons; 2006. https://doi.org/10.1038/npg.els.0005812

25. Tho PT, Byrd JR, McDonough PG. Etiologic and subsequent reproductive performance of 100 couples with a prior history of habitual abortion. Fertil Steril .1979; 32:389-95.
26. Coulam CB. Unexplained recurrent pregnancy loss: epilogue. Clin Obstet Gynecol. 1986; 29; 999-1004.

27. Ke RW. Endocrine basis for recurrent pregnancy loss. Obstet Gynaecol Clin North Am. 2014; 41:103-12.

28. Li TC, Spuijbroek MD, Tuckerman E, Anstie B, Loxley M, Laird S. Endocrinological and endometrial factors in recurrent miscarriage. BJOG. 2000; 107(12):147.

29. Lessey BA, Fritz MA. Defective luteal function. In: Fraser JS, Jansen RP, Lubo RA, Whitehead MI, editors. Estrogens and progestogens in clinical practice. Philadelphia: W.B. Saunders; 1998. p. 437-53.

30. Practice committee of American Society for Reproductive Medicine. Current clinical irrelevance of luteal phase deficiency: a committee opinion. FertilSteril. 2015; 103(4): e27-e32.

31. Kutteh WH, Rote NS, Silver R. Antiphospholipid antibodies and reproduction: the antiphospholipid antibody syndrome. Am J Reprod Immunol. 1999; 41:133-52.

32. Kutteh WH, Hinote CD. Antiphosphlipid antibody syndrome. Obstet Gynecol Clin N Am. 2014; 41: 113-32.

33. Alarcon- Segovia D, Perez-Vasquez ME, Villa AR, Drenkard C, Cabiedes J. Preliminary classification criteria for antiphospholipid syndrome with systemic lupus erythematosus. Semin Arthritis Rheum.1992; 21(5): 275-86.

34. Petri M, Rheinschmidt $M$, Whiting-O'keefe Q, Hellmann D, Corash L. The frequency of lupus anticoagulant in systemic lupus erythematosus: A study of sixty consecutive patients by activated partial thromboplastin time, russell viper venom time, and anticardiolipin antibody level. Ann Intern Med 1987; 106:524-31

35. Donders GG, Bulck BV, Caudron J, Londers L, Vereecken A, Spitz B. Relationship of bacterial vaginosis and mycoplasmas to the risk of spontaneous abortion. Am J Obstet Gynaecol. 2000;183: 431-7.

36. Nigro G, Mazzocco M, Mattia E Carlo di RG, Carta G, Anceschi MM. Role of the infections in recurrent spontaneous abortion. J Matern Fetal Neonatal Med. 2011; 24: 983-9.

37. Dar L, Pati S, Patro A, Deorari AK, Rai SK, Kant S, et al. Congenital cytomegalovirus infection in a highly seropositive semi-urban population in India. Pediatr Infect Dis J. 2008; 27: 841-3. 
The New Indian Journal of OBGYN. 2021 (January-June);8(2)

38. Quenby SM, Farquharson RG. Predicting recurrent miscarriage: What is important? Obstet Gynaecol.1993; 82:132-8.

39. Saravelos SH, Regan L. Unexplained recurrent pregnancy loss. Obstet Gynaecol Clin N Am. 2014; 41: 157-66.

Sukhbir Pal Kaur ${ }^{1}$, Susmita Sharma ${ }^{2}$, Gaganlata ${ }^{3}$

${ }^{1}$ Associate Professor, Department of Obstetrics and Gynaecology, MMIMSR (deemed to be University) Mullana, Ambala, Haryana, India; ${ }^{2}$ Professor, Department of Obstetrics and Gynaecology, Adesh Medical College and Hospital, Mohri, Shahbad, Haryana, India; ${ }^{3}$ Assistant Professor, Department of Obstetrics and Gynaecology, Adesh Medical College and Hospital, Mohri, Shahbad, Haryana, India. 